\title{
A Two-Stage Approach to Harmonic Rejection Mixing Using Blind Interference Cancellation
}

\author{
N. A. Moseley, Student Member, IEEE, E. A. M Klumperink, Member, IEEE, and B. Nauta, Fellow, IEEE
}

\begin{abstract}
Current analog harmonic rejection mixers typically provide 30-40 dB of harmonic rejection, which is often not sufficient. We present a mixed analog-digital approach to harmonic rejection mixing that uses a digital interference canceler to reject the strongest interferer. Simulations indicate that, given a practical RF scenario, the digital canceler is able to improve the signal-to-interference ratio by $30-45 \mathrm{~dB}$.
\end{abstract}

Index Terms-Adaptive filter, CMOS, cognitive radio, gain and phase mismatch, harmonic rejection mixing, I/Q imbalance, interference canceling, LMS, software-defined radio, switching mixers.

\section{INTRODUCTION}

$\mathbf{S}$ WITCHING mixers have good high-level signal behaviour but suffer from harmonic downmixing. RF signals present at multiples of the local oscillator $(\mathrm{LO})$ frequency $\omega_{\mathrm{LO}}$, termed harmonic images, will be down-mixed to baseband thus causing interference to the desired signal. This lack of harmonic rejection (HR) needs to be addressed in the design of receivers.

In traditional single-band direct-conversion receiver frontends, a fixed high-order RF filter is sufficient to attain good HR. Multiband receivers require a tracking RF filter or multiple RF filters. Such RF tracking filters typically consume a lot of power [1] and take up much die or board space, which makes both solutions unattractive for CMOS integration.

The requirements of the RF filters are exacerbated by the use of square waves as the LO waveform or the use of switching mixers in the frontend [2]. In a direct-conversion receiver, the (baseband) output of the mixer is the convolution in the frequency domain of the effective LO waveform spectrum with the $\mathrm{RF}$ spectrum at the antenna. Thus, the harmonic content of the effective $\mathrm{LO}$ waveform determines which frequencies are mixed down to baseband. As the effective LO waveform contains many strong harmonics, the mixer offers less HR compared to a multiplier with a sinusoidal LO. Still, square waves are preferred over sinusoids, especially for flexible spectrum access [2], because the former are easier to produce over a wide frequency range in an IC using digital circuits. Unwanted mixing products, such as the third and fifth harmonic images, can be cancelled by using multiple mixers. Each mixer is driven by a $\mathrm{LO}$ square wave of different duty cycle. Their outputs are combined in a weighted sum to form the final output. Provided the mixers operate linearly from RF to IF, this parallel mixer structure may

Manuscript received January 21, 2008; revised April 1, 2008. Current version published October 15, 2008. This paper was recommended by Associate Editor B. Razavi.

The authors are with the IC-Design Group, CTIT, University of Twente, 7500 AE, Enschede, The Netherlands (e-mail: n.a.moseley@utwente.nl).

Digital Object Identifier 10.1109/TCSII.2008.926796

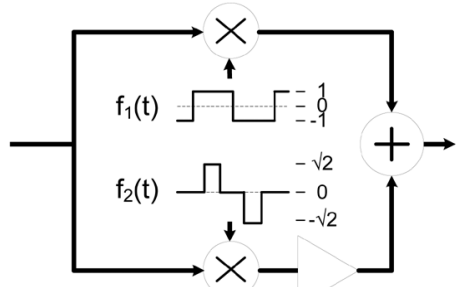

Parallel switching mixers (all paths are differential)

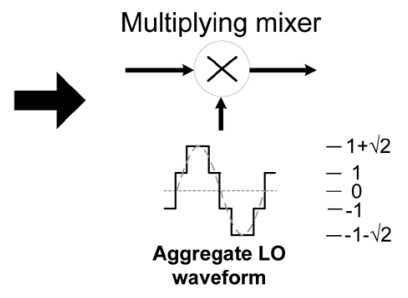

System model
Fig. 1. Modeling a set of parallel mixers as a single mixer. The single mixer is driven by the aggregate effective $\mathrm{LO}$ waveform.

be treated as a single mixer from a system point-of-view. The effective aggregate LO waveform, is simply the weighted sum of the individual LO waveforms, see Fig. 1.

The duty cycles and weights of each LO waveform are chosen so that the aggregate LO waveform contains a reduced set of harmonics. The more mixing paths are introduced, the less harmonics are present in the aggregate waveform. In practice, the LO signals are generated using a multi-phase clock generator. This technique has been successfully applied in CMOS transmitters [3] and in receivers [4], [5]. In [4], the average harmonic rejection across 10 chips is around $40 \mathrm{~dB}$ and in [5] the HR ratio is around $42 \mathrm{~dB}$. This is often not enough. Suppose you want to receive a TV signal at $900 / 3=300 \mathrm{MHz}$. A nearby GSM transmitter at about $900 \mathrm{MHz}$ can produce up to $0 \mathrm{dBm}$ at the antenna. It will completely corrupt the TV signal.

In this paper, we present a new two-stage HR concept with a focus on the system level. In addition to HR in the analog domain, we obtain additional HR in the digital domain. A digital solution was chosen because of the need for high-accuracy signal manipulation. Our method requires a second observation of the complex baseband signal in order to reduce the interference caused by the residual harmonic images. This observation is produced by means of two analog subtractors and two additional ADCs.

In Section II, we will introduce a system model of a single mixer. In Section III, the extended multipath mixer is explained. Section IV explains the digital algorithm. This is followed by Section V, which presents our simulation results. Finally, Section VI offers the conclusions.

\section{MiXeRs-A SYSTEM MOdel}

The (differential) mixer shown in Fig. 2 is driven by a square wave $f(t)$. The input $x(t)$ is multiplied by the LO $f(t)$ to form 


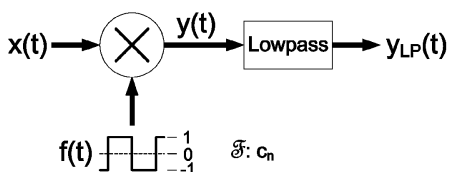

Fig. 2. Block diagram of a mixer with a square wave LO.

the output $y(t)$. Consider an RF input signal $x(t)$ consisting of $n=1 \ldots N$ baseband-equivalent signals $z_{n}(t)$ :

$$
\begin{aligned}
x(t) & =\sum_{n=1}^{N} \Re\left\{z_{n}(t) \cdot e^{j \omega_{\mathrm{LO}} n t}\right\} \\
& =\frac{1}{2} \sum_{n=1}^{N}\left(z_{n}(t) \cdot e^{j \omega_{\mathrm{LO}} n t}+z_{n}^{*}(t) \cdot e^{-j \omega_{\mathrm{LO}} n t}\right)
\end{aligned}
$$

where $\Re$ denotes the real part, $\omega_{\mathrm{LO}}$ is the LO frequency in $\mathrm{rad} / \mathrm{s}$ and * denotes the complex conjugate. The RF signal corresponding to $z_{n}(t)$ can be found at a multiple of the LO frequency, namely $n \cdot \omega_{\mathrm{LO}}$. Also consider the LO signal $f(t)$ as its Fourier series:

$$
f(t)=c_{0}+\sum_{n=1}^{\infty}\left(c_{n}^{*} \cdot e^{-j \omega_{\mathrm{LO}} n t}+c_{n} \cdot e^{j \omega_{\mathrm{LO}} n t}\right)
$$

where $c_{n}$ are the Fourier series coefficients of the LO waveform.

The output after the lowpass filter $y_{L P}(t)$ is calculated by multiplying (1) by (2) and removing the high-frequency components:

$$
y_{L P}(t)=\frac{1}{2} \sum_{n=1}^{\infty}\left(c_{n}^{*} \cdot z_{n}(t)+c_{n} \cdot z_{n}^{*}(t)\right)
$$

Equation (3) shows that the strength of the harmonic images down mixed to baseband depend on the Fourier series of the LO waveform. For instance, when the 3rd harmonic is absent from the LO waveform $\left(c_{3}=0\right)$, the third harmonic image $z_{3}(t)$, found at an RF frequency of $3 \omega_{\mathrm{LO}} \mathrm{rad} / \mathrm{s}$, will not be present in the baseband signal $y_{\mathrm{LP}}(t)$.

\section{A QUADRATURE HARMONIC-REJECTION MIXER}

Consider the harmonic-rejection mixer block diagram shown in Fig. 3. The RF signal present at the antenna passes through an RF filter after which it is distributed across four mixers. The top two mixers produce the in-phase channel $r_{I}(t)$ while the bottom two mixers produce the quadrature channel $r_{Q}(t)$. For now, we will ignore $v_{I}(t)$ and $v_{Q}(t)$.

The Fourier series coefficients of the aggregate LO waveform of the I-channel, $\alpha_{n}$, and those of the Q-channel, $\beta_{n}$, can be expressed as functions of the Fourier series coefficients of the mixer LO waveforms $f_{1, I}(t), f_{2, I}(t), f_{1, Q}(t)$ and $f_{2, Q}(t)$, which are $c_{1, n}, c_{2, n}, d_{1, n}$, and $d_{2, n}$ respectively. By inspection of Fig. 3, $r_{I}(t)$ and $r_{Q}(t)$ are found to be:

$$
\begin{aligned}
& r_{I}(t)=\frac{1}{2} \sum_{n=1}^{\infty}\left(\alpha_{n}^{*} \cdot z_{n}(t)+\alpha_{n} \cdot z_{n}^{*}(t)\right) \\
& r_{Q}(t)=\frac{1}{2} \sum_{n=1}^{\infty}\left(\beta_{n}^{*} \cdot z_{n}(t)+\beta_{n} \cdot z_{n}^{*}(t)\right),
\end{aligned}
$$

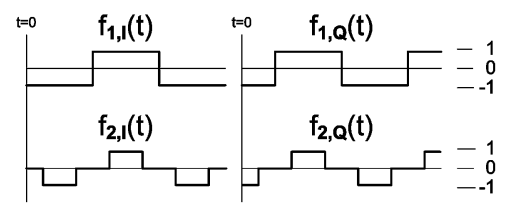

Fig. 4. Effective LO waveforms of the I-channel $\left(f_{1, I}(t), f_{2, I}(t)\right)$ and the Q-channel $\left(f_{1, Q}(t), f_{2, Q}(t)\right)$.

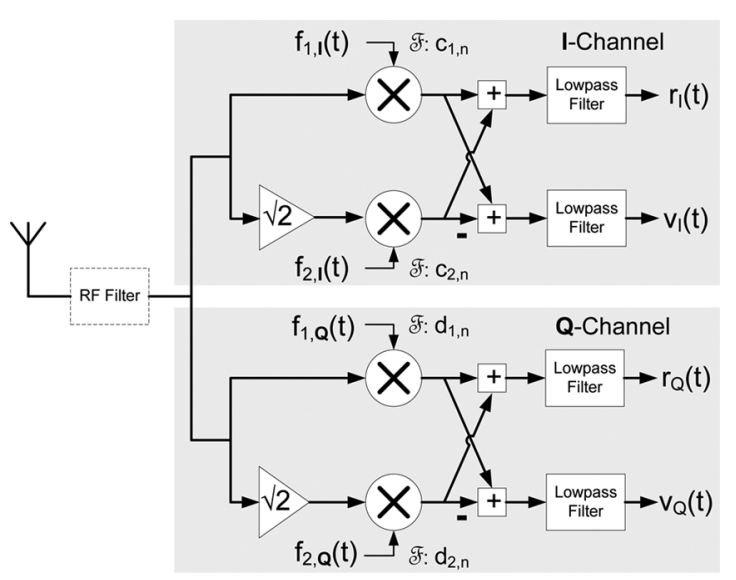

Fig. 3. Quadrature harmonic rejection mixer topology. The LO waveforms are denoted by $f_{1, I}(t), f_{2, I}(t), f_{1, Q}(t)$ and $f_{2, Q}(t)$. Their Fourier series coefficients are $c_{1, n}, c_{2, n}, d_{1, n}$ and $d_{2, n}$ respectively.

where $\alpha_{n}=c_{1, n}+\sqrt{2} c_{2, n}$ and $\beta_{n}=d_{1, n}+\sqrt{2} d_{2, n}$. Thus, the complex baseband output $r(t)=r_{I}(t)+j \cdot r_{Q}(t)$ is equal to

$$
r(t)=\frac{1}{2} \sum_{n=1}^{\infty}\left[\left(\alpha_{n}-j \beta_{n}\right)^{*} \cdot z_{n}(t)+\left(\alpha_{n}+j \beta_{n}\right) \cdot z_{n}^{*}(t)\right] .
$$

Given the LO waveforms shown in Fig. 4, it can be shown that $\alpha_{n}=0$ and $\beta_{n}=0$ when

$$
n \in\{3+8 k, 5+8 k \mid k \in(\mathbb{N} \cup\{0\})\}
$$

or when $n$ is even. As a result, the even-order, third and fifth harmonic images are rejected. The first uncancelled harmonic image is the seventh. Thus, the complex baseband output $r(t)$ only contains the desired signal assuming that the RF filter at the antenna removes the seventh and higher harmonic images. The RF filter requirements are greatly relaxed compared to a singlemixer case where the filter would have to remove the third and higher harmonic images, assuming a perfect differential system.

We will not consider analog mixer circuit details as our main focus is on the concept. In fact, there are many ways to implement the scheme mentioned, e.g., weighted Gilbert cells [3].

\section{A. Achievable Harmonic Rejection Ratio}

Unfortunately, complete rejection of the harmonic images as specified by (5) through the principle outlined above, only applies when the LO waveforms $f_{1, I}(t), f_{2, I}(t), f_{1, Q}(t)$ and $f_{2, Q}(t)$ are exactly as specified in [3].

In practice, the LO waveforms are derived from an eightphase clock. Such a multi-phase clock has dynamic and static timing errors. These errors reduce the amount of HR. In [6] it is 
shown that static timing errors are typically dominant over dynamic timing errors.

Assuming the multi-phase clock frequency is exact, seven out of the eight clock edges can have static timing errors, $\tau_{1 . .7}$, as there are seven degrees of freedom. A second source of error that limits the HR ratio is a deviation in the required $1: \sqrt{2}$ ratio between the paths, see Fig. 3. A perfect $1: \sqrt{2}$ ratio is hard to achieve in hardware owing to component mismatches and the fact that $1: \sqrt{2}$ is an irrational ratio.

In addition to limiting the achievable $H R$, the aforementioned errors also cause I/Q imbalance. There exist many solutions to the I/Q imbalance problem and we will therefore not cover I/Q imbalance here. Instead, we refer to [7], [8] and the references therein.

\section{B. Improving the Harmonic Rejection}

The harmonic rejection ratio (HRR) can be improved by admittance scaling [6], [9] the clock generator and mixer circuits at the cost of increased power consumption. Scaling the admittance of a circuit by a factor $\lambda$ decreases the amplitude mismatch by a factor $\sqrt{\lambda}$ and increases its power consumption by a factor $\lambda$. The same holds for timing mismatch errors. However, increasing the HR performance considerably, e.g., from 40 to greater than $70 \mathrm{~dB}$, through admittance scaling (i.e., wider transistors) would increase the power consumption from milliwatts to watts, which is clearly undesirable.

A second approach to improve the HRR is to calibrate the timing of the multi-phase clock using analog techniques. This solution requires many calibration points within the circuit as each clock edge must be controlled. A purely digital HRR-enhancement technique is preferable because it is more scalable, easier to migrate to a new IC process and it can be designed independently from the analog front-end. We present such a technique based on interference cancellation in Section IV.

\section{AN InTERfERENCE CANCELlation TeChNiQue FoR ADDITIONAL HARMONIC REJECTION}

Interference cancellation techniques based on LMS adaptive filtering have been used in a wide variety of situations, such as active noise canceling [10], beam forming and radar [11]. The concept is to subtract an estimate of the interferer from the contaminated desired signal. This estimate is scaled and rotated by an adaptive filter to remove any phase or amplitude difference between the interference in the two paths.

In our application, the contaminated desired signal is $r(t)$ and the estimate of the interference is $v(t)=v_{I}(t)+j \cdot v_{Q}(t)$. The signal $v(t)$ is produced by subtracting instead of adding the paths as is shown in Fig. 3. The signal $v(t)$ can be expressed as

$$
v(t)=\frac{1}{2} \sum_{n=1}^{\infty}\left[\left(\delta_{n}-j \gamma_{n}\right)^{*} \cdot z_{n}(t)+\left(\delta_{n}+j \gamma_{n}\right) \cdot z_{n}^{*}(t)\right]
$$

where $\delta_{n}=c_{1, n}-\sqrt{2} c_{2, n}$ and $\gamma_{n}=d_{1, n}-\sqrt{2} d_{2, n}$.

The signals that are attenuated in $r(t)$ are not attenuated in $v(t)$ and vice versa. So, $v(t)$ forms an estimate of the interference as the desired signal is attenuated, while the interference is not.

From (4), it is clear that the desired signal contains both the signals $z_{n}(t)$ and their complex conjugates $z_{n}^{*}(t)$. In order to

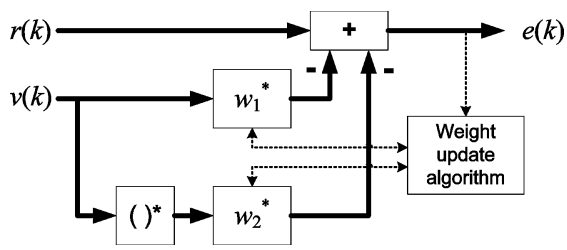

Fig. 5. Digital harmonic rejection structure.

reject the harmonic images, both $z_{n}(t)$ and $z_{n}^{*}(t)$ for $n>1$ must be canceled by the interference canceler. Thus, we must apply two-input MISO interference canceling [12], [13], where $v(t)$ and $v^{*}(t)$ serve as the two inputs.

Before we can supply the digital interference canceler with the signals $v(t)$ and $r(t)$, we must convert them to the digital domain using two analog-digital converters (ADCs) with complex-valued inputs.

\section{A. From Analog to Digital}

The two complex analog signals $r(t)$ and $v(t)$ are sampled by four ADCs. The ADCs share a common clock and are therefore synchronized. Assuming that the anti-aliasing filters in front of the ADCs are perfect, we have two complex discrete-time signals $\bar{r}(k)$ and $\bar{v}(k)$, which are defined as

$$
\bar{r}(k)=r\left(k \tau_{\text {sample }}\right) \quad \bar{v}(k)=v\left(k \tau_{\text {sample }}\right)
$$

where $\tau_{\text {sample }}$ is the time (in seconds) between each sample of the ADCs and $k$ is the sample index.

We will simply write $r(k)$ and $v(k)$ instead of $\bar{r}(k)$ and $\bar{v}(k)$ from this point onward as the remainder of the paper focuses on discrete-time digital signal processing only.

The quantization noise in $\bar{v}(k)$ needs to be lower than the desired additional HR achieved by the digital part. The additional ADCs, used to sample $v(k)$, require at least $\lceil S / 6\rceil$ bits, where $\mathrm{S}$ is the minimum desired suppression in $\mathrm{dB}$ and $\lceil$.$\rceil denotes$ rounding up to the nearest integer.

\section{B. Digital Interference Canceler}

Fig. 5 shows the proposed discrete-time HR structure. It comprises two one-tap complex finite-impulse response (FIR) filters, a complex-conjugate operator block (to produce $v^{*}(k)$ ) and an adder with two negating inputs. The filter weights $w_{1}$ and $w_{2}$ rotate and scale $v(k)$ and $v^{*}(k)$ such that the phase and amplitude of the strongest harmonic image lines up with the same harmonic image in $r(k)$. In effect, the power of the strongest harmonic image is greatly reduced in the output signal $e(k)$. The output $e(k)$ can be written as

$$
e(k)=r(k)-w_{1}^{*} \cdot v(k)-w_{2}^{*} \cdot v^{*}(k)
$$

As each harmonic image in $v(k)$ has a different phase and amplitude with respect to $r(k)$, each harmonic image requires a different value of $w_{1}$ and $w_{2}$ to cancel. In effect, the interference canceler can reject only one harmonic image. This may seem like a big disadvantage but the probability that more than one very strong harmonic image is present at RF is quite low. Also consider the fact that the analog part already provides $40 \mathrm{~dB}$ of 
rejection and that this does not include the additional rejection offered by an RF filter.

\section{Obtaining the Filter Weights $w_{1}$ and $w_{2}$}

The filter weights $w_{1}$ and $w_{2}$ are estimated using the LMS adaptive filter algorithm [13]. This algorithm was chosen for its simple implementation in hardware, its robustness and the fact that the convergence conditions are well understood.

The optimum least-squares filter weights $\mathbf{w}_{\text {opt }}$ are obtained by minimizing the following cost function [13] with respect to w:

$$
J(\mathbf{w})=E\left\{|r(k)|^{2}-\mathbf{w}^{H} \mathbf{p}-\mathbf{p}^{H} \mathbf{w}+\mathbf{w}^{H} \mathbf{R} \mathbf{w}\right\}
$$

where

$$
\begin{aligned}
& \mathbf{w}=\left[\begin{array}{c}
w_{1} \\
w_{2}
\end{array}\right] \quad \mathbf{p}=\left[\begin{array}{c}
E\left\{v(k) r^{*}(k)\right\} \\
E\left\{v^{*}(k) r^{*}(k)\right\}
\end{array}\right] \\
& \mathbf{R}=\left[\begin{array}{cc}
E\left\{|v(k)|^{2}\right\} & E\left\{v^{2}(k)\right\} \\
E\left\{\left(v^{*}(k)\right)^{2}\right\} & E\left\{|v(k)|^{2}\right\}
\end{array}\right]
\end{aligned}
$$

$H$ denotes the Hermitian operator and $E\{$. $\}$ denotes the expectation operator.

Note that $\mathbf{p}$ and $\mathbf{R}$ can be expressed as functions of the $\alpha_{n}$, $\beta_{n}, \delta_{n}$ and $\gamma_{n}$ coefficients. Due to limited article space, this is left as an exercise for the reader. As the coefficients depend on the LO static timing errors and mismatches in the $1: \sqrt{2}$ ratio, the algorithm is able to compensate for both.

The LMS adaptive filter algorithm [13] minimizes (8) without requiring explicit knowledge of the statistics in (9) and (10) by using the following filter update rule:

$$
\hat{\mathbf{w}}(k+1)=\hat{\mathbf{w}}(k)+\mu \mathbf{v}(k) e^{*}(k)
$$

where the learning coefficient $\mu<\left(E\left\{|v(k)|^{2}\right\}\right)^{-1}$ for stability, $\hat{\mathbf{w}}(k)$ is an estimate of $\mathbf{w}_{\text {opt }}$ at time $k$ and

$$
\mathbf{v}(k)=\left[\begin{array}{c}
v(k) \\
v^{*}(k)
\end{array}\right] .
$$

The algorithm requires a total of four complex multiplies, four complex add/subtracts and two complex-times-scalar multiplies per sample, which is negligble compared to the remaining digital baseband operations, such as channel selection, synchronization and equalization. Simulations were performed to evaluate the HR performance of the algorithm.

\section{Simulations}

The LMS adaptation coefficient $\mu$ is chosen to be $10^{-4}$. $E\left\{|v(k)|^{2}\right\}^{-1}$. This proved to be a good tradeoff between convergence time, which is around 30.000 samples, and accuracy.

In order to verify that our algorithm works, we performed a simulation of the HR mixer and compensator. The static timing errors $\tau_{1 . .7}$ of the multi-phase clock generator are drawn from a zero mean gaussian distributed process with a standard deviation equal to $0.5 \%$ of the aggregate LO period. The $1: \sqrt{2}$ ratio was approximated by 1:1.4. This results in HR figures from 30 to $40 \mathrm{~dB}$ for the analog part. The simulations do not include an RF filter.

In the first simulation, five sinusoidal signals (the desired and four harmonic images) are present at the antenna. Their base-
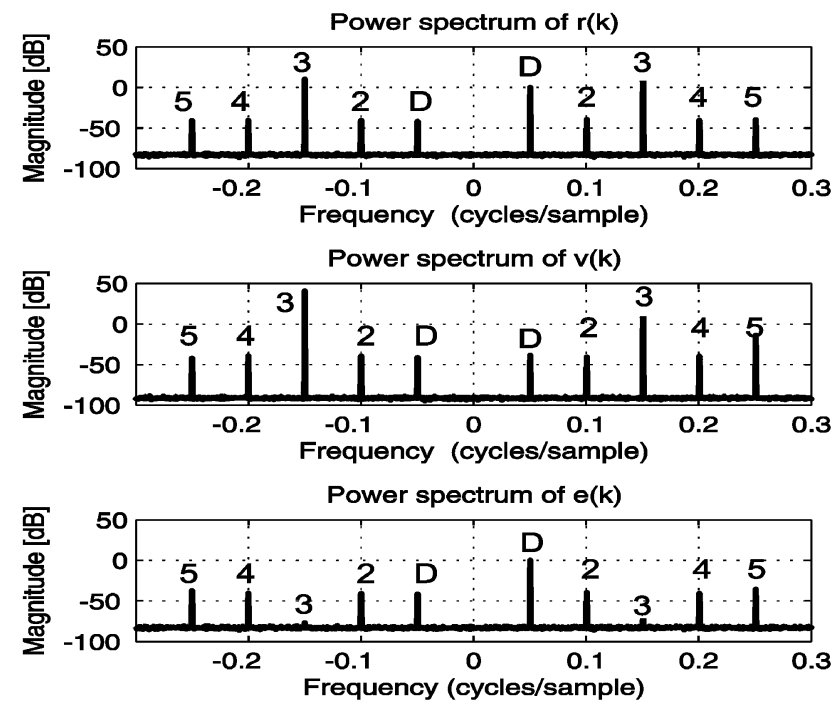

Fig. 6. Baseband spectra of $r(k), v(k)$ and $e(k)$. The baseband-equivalent desired signal has unit variance $\sigma_{d}=1$ at the antenna. The remaining RF signal strengths are: $3 \mathrm{rd}=50 \mathrm{~dB} .2 \mathrm{nd}, 4 \mathrm{th}, 5 \mathrm{th}=0 \mathrm{~dB}$ w.r.t the desired signal. The signals are indicated by ' $\mathrm{D}^{\prime}, 2^{\prime}, 3^{\prime},{ }^{\prime} 4$ ' and ' 5 ', respectively. All signals are sinusoidal. The baseband frequencies of the signals are $0.05,0.10,0.15,0.20$, and 0.25 cycles/sample. The ADCs have an infinite number of bits.

band frequencies are $0.05,0.10,0.15,0.20$ and 0.25 cycles/ sample, respectively. Their signal strengths are $0 \mathrm{~dB}$ with respect to the unit-variance desired signal $\left(\sigma_{\text {desired }}^{2}=1\right)$, except for the $3 \mathrm{rd}$ harmonic image, which is $50 \mathrm{~dB}$. This scenario represents a typical case where there is one very strong interferer and several weaker ones. Complex AWG noise $\sigma_{\text {noise }}^{2}=10^{-5}$ is added to each baseband-equivalent signal to simulate RF noise. This figure was chosen low to be able to show clear signal spectra. The ADCs have an infinite number of bits.

Fig. 6 shows the spectra of the mixer output $r(k)$, the interference output $v(k)$ and the compensated output $e(k)$. From the $r(k)$ spectrum, it is clear that the HR offered by the analog mixer is not sufficient. The 3rd harmonic image is $10.4 \mathrm{~dB}$ stronger that the desired signal. The $v(k)$ signal indeed estimates the interference: the desired signal is suppressed while the $3 \mathrm{rd}$ and 5 th harmonic images are boosted.

The dominating signal in the compensated signal $e(k)$ is the desired signal, as shown by its spectrum in Fig. 6. The third harmonic image is found at $-76.9 \mathrm{~dB}$, which is a decrease of $87.3 \mathrm{~dB}$. As the other harmonic images are not rejected, the final signal-to-interference ratio will be determined by these images.

To show the systems works with a modulated signal and realistic ADC quantization errors, the desired signal is changed to a random 16-QAM signal centered at $\omega_{\mathrm{LO}}$ and a bandwidth equal to the baseband bandwidth. Given $35 \mathrm{~dB}$ of analog $\mathrm{HR}$ and $50 \mathrm{~dB}$ stronger interference, we require $35 \mathrm{~dB}$ of digital $\mathrm{HR}$ for a 20-dB SNR so 6 bits are needed for the additional ADCs. The main ADCs use 10 bits. The constellation diagrams of $r(k)$ (left) and $e(k)$ (right) are shown in Fig. 7. The constellation plot of $e(k)$ clearly shows a 16-QAM constellation, while the $r(k)$ plot does not. As ADC power scales with $2^{\text {bits }}$, the additional ADCs add an insignificant amount of power to the system while they improve the total HR performance greatly.

The signal-to-interference ratio (SIR) after compensation depends on the RF scenario, the $1: \sqrt{2}$ ratio errors and the timing 

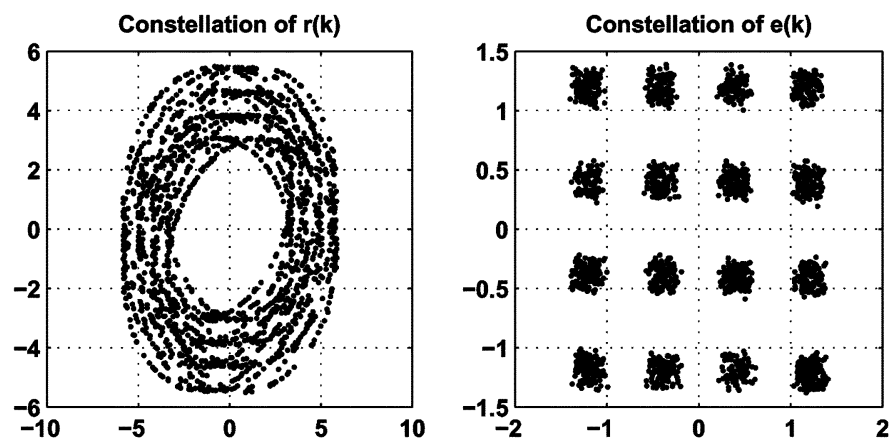

Fig. 7. Constellation diagrams of $r(k)$ and $e(k)$. The baseband-equivalent desired signal is 16-QAM with unit variance $\sigma_{d}=1$ at the antenna. The remaining sinusoidal RF signal strengths are: $3 \mathrm{rd}=50 \mathrm{~dB}$. $2 \mathrm{nd}$, 4 th, 5 th $=0 \mathrm{~dB}$ w.r.t. the desired signal. The ADCs use 10 and 6 bits for $r(k)$ and $v(k)$ respectively.
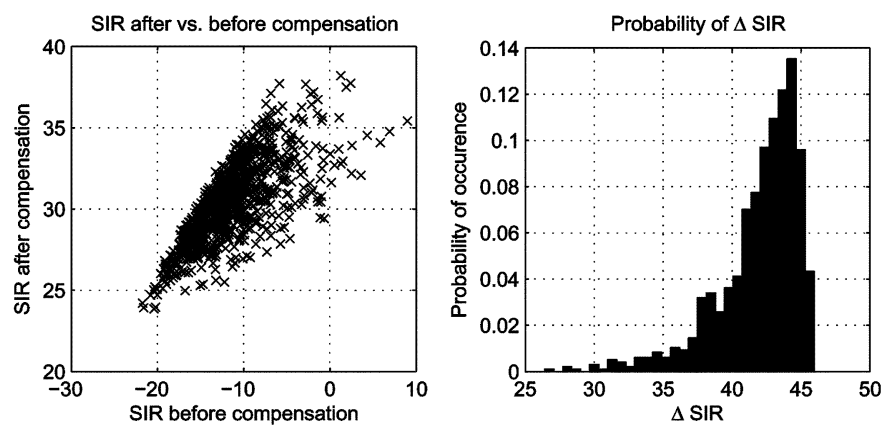

Fig. 8. Left: a scatter plot of the SIR after vs. before compensation. Right: a histogram showing the probability of a certain SIR improvement caused by the compensator.

errors $\tau_{1 . .7}$. Therefore, a simple performance estimate of the SIR is not tractable. However, an indication of the compensator's performance can be given in the form of a SIR scatter plot and its distribution, as shown in Fig. 8. The plots were produced by taking 1000 different realizations of $\tau_{1 . .7}$ and determining the SIR of $r(k)$ and $e(k)$ for each realization. The RF scenario was kept the same as used in Fig. 6 and the ADCs use an infinite number of bits. From the histogram it is clear that the compensation structure provides an increase of more than $36 \mathrm{~dB}$ in $90 \%$ of the cases. The mean SIR at the output $e(k)$ is $30.3 \mathrm{~dB}$, an increase of $41.9 \mathrm{~dB}$.

A strong even-order harmonic image can also be compensated, although not as effectively as the 3rd or the 5th because even-order harmonic images are suppressed in the interference estimate $v(n)$. This can be improved if a better estimate of the even-order harmonic interference is used together with two extra 1-tap filters.

\section{CONCLUSIONS}

We have presented a method to increase the harmonic rejection (HR) of an analog HR mixer by means of an adaptive compensation structure. The structure comprises two complex 1-tap FIR filters and a complex conjugation block. The filter weights are obtained by the LMS adaptive filter algorithm.

The proposed method is able to reject the strongest harmonic image. The final signal-to-interference ratio (SIR) at the output depends strongly on the static timing errors of the aggregate LO waveform and RF signals present at the antenna. Therefore, it is intractable to provide a performance figure valid for all cases. The effectiveness of our solution is shown by means of statistical simulation: a SIR increase of more than $36 \mathrm{~dB}$ is found in $90 \%$ of the cases. The mean SIR increase is $41.8 \mathrm{~dB}$.

\section{REFERENCES}

[1] J. R. Tourret et al., "Sip tuner with integrated LC tracking filter for both cable and terrestrial TV reception," IEEE J. Solid-State Circuits, vol. 42, no. 12, pp. 2809-2821, Dec. 2007.

[2] E. Klumperink, R. Shrestha, E. Mensink, V. Arkesteijn, and B. Nauta, "Cognitive radios for dynamic spectrum access-Polyphase multipath radio circuits for dynamic spectrum access," IEEE Commun. Mag., vol. 45, no. 5, pp. 104-112, May 2007.

[3] J. A. Weldon, R. S. Narayanaswami, J. C. Rudell, L. Lin, M. Otsuka, S. Dedieu, L. Tee, K.-C. Tsai, C.-W. Lee, and F. P. R. Gray, "A $1.75-\mathrm{GHz}$ highly integrated narrow-band CMOS transmitter with harmonic-rejection mixers," IEEE J. Solid-State Circuits, vol. 36, no. 12, pp. 2003-2015, Dec. 2001.

[4] Z. Ru, E. A. M. Klumperink, and B. Nauta, "A discrete-time mixing receiver architecture with wideband harmonic rejection," in IEEE ISSCC Dig. Tech. Papers, 2008.

[5] M. Gupta, S. Lerstaveesin, D. Kang, and B.-S. Song, "A 48-to-860 $\mathrm{MHz}$ CMOS direct-conversion TV tuner," in IEEE ISSCC Dig. Tech. Papers, 2007.

[6] R. C. H. van der Beek, E. A. M. Klumperink, C. S. Vaucher, and B. Nauta, "Low-jitter clock multiplication: A comparison between PLLs and DLLs," IEEE Trans. Circuits Syst. II, Analog Digital Signal Process., vol. 49, no. 8, pp. 555-566, Aug. 2002.

[7] M. Valkama, M. Renfors, and V. Koivunen, "Advanced methods for I/Q imbalance compensation in communication receivers," IEEE Trans. Signal Process., vol. 49, no. 10, pp. 2355-2344, Oct. 2001.

[8] M. Windisch and G. Fettweis, "Blind estimation and compensation of I/Q imbalance in OFDM receivers with enhancements through Kalman filtering," in IEEE/SP 14th Workshop on Statistical Signal Process. 2007 (SSP '07), Aug. 26-29, 2007, pp. 754-758.

[9] E. A. M. Klumperink and B. Nauta, "Systematic comparison of HF CMOS transconductors," IEEE Trans. Circuits Syst. II, vol. 50, pp. 728-741, Oct. 2003.

[10] S. M. Kuo and D. R. Morgan, Active Noise Control Systems. Hoboken, NJ: Wiley Interscience, 1996.

[11] R. N. Ghose, Interference Mitigation. Piscataway, NJ: IEEE Press, 1996.

[12] Y. Huang, J. Benesty, and J. Chen, Acoustic MIMO Signal Processing. New York: Springer, 2006.

[13] S. Haykin, Adaptive Filter Theory. Upper Saddle River, NJ: Prentice Hall, 2002. 